\title{
Global-Local View: Scalable Consistency for Concurrent Data Types
}

\author{
Deepthi Devaki Akkoorath ${ }^{1}$, José Brandão ${ }^{3}$, Annette Bieniusa ${ }^{2}$, \\ and Carlos Baquero ${ }^{4}$ \\ ${ }^{1}$ Technical University of Kaiserslautern, Kaiserslautern, Germany, \\ akkoorath@cs.uni-kl.de \\ ${ }^{2}$ Technical University of Kaiserslautern, Kaiserslautern, Germany, \\ bieniusa@cs.uni-kl.de \\ ${ }^{3}$ Universidade do Minho, Braga, Portugal, \\ pg30467@alunos.uminho.pt \\ ${ }^{4}$ HASLab, Universidade do Minho \& INESC TEC, Braga, \\ Portugal, cbm@di.uminho.pt
}

November 8, 2018

\begin{abstract}
Concurrent linearizable access to shared objects can be prohibitively expensive in a high contention workload. Many applications apply ad-hoc techniques to eliminate the need of synchronous atomic updates, which may result in non-linearizable implementations. We propose a new programming model which leverages such patterns for concurrent access to objects in a shared memory system. In this model, each thread maintains different views on the shared object - a thread-local view and a global view. As the thread-local view is not shared, it can be updated without incurring synchronization costs. These local updates become visible to other threads only after the thread-local view is merged with the global view. This enables better performance at the expense of linearizability. We show that it is possible to maintain thread-local views and to perform merge efficiently for several data types and evaluate their performance and scalability compared to linearizable implementations. Further, we discuss the consistency semantics of the data types and the associated programming model.
\end{abstract}

\section{Introduction}

Concurrent programming on shared-memory architectures is notoriously difficult. A concurrent system consists of a set of processes communicating implicitly through shared data structures. The visibility of updates on these data structures depends on the intricate interplay of synchronization mechanisms as defined by the memory model. Linearizability [10] has turned out to be a fundamental notion on simplifying the reasoning about correctness of shared data 
structures for programmers. This consistency model formalizes the notion of atomicity for high-level operations. In an execution, every method call is associated with a linearization point, a point in time between its invocation and its response. The call appears to occur instantaneously at its linearization point, behaving as specified by the sequential definition.

While linearizability is very useful for reasoning about the correctness of concurrent data structures, its implementation can be prohibitively expensive. As the number of cores increases in a multi-core system, the synchronization cost becomes more apparent that, it favors the relaxation of the concurrent objects semantics for scaling the programs [17]. In practice, programming patterns are emerging that attempt to limit the associated cost of the required synchronization on the memory accesses. For example, in the widely-used messaging library ZeroMQ, adding messages to the queue is at the core of the application. While lock-free linearizable queues are fast, the developers observed that enqueuing new messages was affecting the overall performance, especially in high contention workloads [18. However, only the relative order of messages from a single thread are relevant for the semantics of the message queue; it is not necessary to maintain a strict order of enqueue operations when two independent threads try to insert messages concurrently into the queue. To overcome the performance penalty, the developers re-engineered their message queue such that multiple messages are added as a batch, thus using only one single atomic operation.

For another example, consider a shared counter that is concurrently updated by several threads. The final value of the counter must include all increments performed, but the order of increments is not relevant since all increments are commutative. If each increment executed by each thread is an atomic operation made visible to all other threads, it can become a bottleneck limiting the performance of the program [5. In many cases, it is sufficient to execute the increment on some thread-local variable and to apply a combined update to the shared object.

In this paper, we propose a new programming model for shared objects that leverages the different views of an object, the global-local view model. In this model, each thread has a local view of the object which is isolated from other threads. Threads update and read the local view. The local updates, though visible in a local view, are made visible on a global view only after an explicit two-way merge operation is performed. The other threads observe these changes once they synchronize, by merge, their local view with the global view. As the local view is non-shared, the local updates can be executed without requiring synchronization, thus enabling better performance, albeit at the expense of linearizability.

In addition to the local operations, the model also provides synchronous operations on the global view. Consider, for example, a queue where the enqueues have been executed on the local view. To guarantee that the elements are dequeued only once, dequeues are executed atomically on the global view. We call the operations that perform only on local view, weak operations and those on global view, strong operations. Combining operations on the global and the local views, we can build data types with customizable semantics on the spectrum between sequential and purely mergeable data types. Mergeable data types provide only weak and merge operations; hybrid mergeable data types offer both weak and strong operations. An application that uses a hybrid mergeable data 
type may use weak updates when a non-linearizable access is sufficient and can switch to use only strong operations when stronger guarantees are required.

In distributed systems, mergeable data types [16, 7, are already widely in use. In this setting, each replica can be concurrently updated without requiring any synchronization and can later be merged with other replicas, while it is guaranteed that all nodes reach a convergent state once all updates have been delivered. To our knowledge, the applicability of such data types in a multi-core shared-memory setting has not been explored systematically, yet. In previous work, we have demonstrated how such types can be employed in Software Transactional Memory to prevent aborts by resolving conflicts automatically [3]. In another work, Doppel [15], an in-memory multi-core database, uses a per-core replica of objects and type-specific merge operations to parallelize conflicting transactions.

Contributions This paper makes the following contributions:

1. We propose a new programming model, global-local view, for scalable multi-threaded applications that implements an adaptable trade-off between update visibility and synchronization cost (Section 3).

2. We provide a unified operational model of mergeable and hybrid data types and give a formal definition of their consistency semantics (Section 5..

3. We discuss the implementation of a mergeable counter, a hybrid counter, and a hybrid queue (Section 4) and compare their scalability with their linearizable counterparts in both low and high contention workloads (Section 6).

In our preliminary work [4, we propose a mergeable counter and bag implementation. In contrast, this paper explores the concept of mergeability in depth by providing a formal model, specifications of further data types and an experimental evaluation.

\section{Related Work}

Programming models: Maintaining per-thread replicas and performing updates on them has been considered by different programming models in the literature. In Concurrent Revisions [6], a forked thread's state is initially, a copy of its parent thread's state. The forked thread makes changes on its copy which is merged to the parent thread when it is joined back. During the join, conflicting updates are resolved using type-specific merge operations. The focus of this work is on fork-join model, where threads can communicate their state only when they join their parent. In contrast, we provide a generic model for the data types where a two-way merge and strong updates can share states among the threads at any point in the execution, thus enabling the applications to tune their use.

Global Sequence Protocol (GSP) 8 is a model for replicated and distributed data systems. Similar to our model, GSP has a global state which is represented as a sequence of operations. Each client stores a prefix of this global sequence. 
The updates by client are first appended to the local sequence of pending operations and then broadcast to other replicas using a reliable total order broadcast protocol which enforces a single order on the global sequence. Since GSP addresses a distributed system's system model, with no bounds on message delays, there is much less control on replica divergence and liveness of the global sequence evolution. In contrast, here we address a shared-memory concurrent architecture that allows to reason about bounds on divergence and stronger progress guarantees on the evolution of shared state.

Read-copy-update (RCU) [9] is a synchronization mechanism to allow processes to read a shared object while a concurrent modification is in progress. Similar to our model, multiple versions of the object are maintained so that readers observe consistent state while a modification is in progress. However, RCU is suited only for a single writer-multiple readers scenario. Read-log-update (RLU) 13 is an improvement over RCU that allows concurrent writers. Unlike our model, concurrent writes are serializable which is achieved by serializing the writes or by fine-grained locking.

Relaxed consistency models: Many models attempt to relax the strict semantics of linearizability 10 to achieve better performance. Quasi linearizability [1] allows each operation to be linearized at a different point at some bounded distance from its strict linearization point. For example, a queue that dequeues in a random order, but never returns empty if the queue is not empty, is a quasi linearizable queue. Quasi linearizability, thus allows more parallelism by allowing flexible implementations. Our work is complimentary to this model, allowing a flexible combination of strong and weak updates to achieve different consistency semantics. Weak and medium future linearizability [11 is applicable to the data types implemented using futures which allow flexible reordering of the operations. Others models, such as k-linearizability 2] and quiescent consistency [19], also define the correctness based on some sequential history, possible reordered, of the operations.

Mergeable Data Types: The idea of concurrent updates to the replicas of an object and merging them to a convergent state was formalized by Conflict free Replicated Data Types (CRDTs) [16], which are now widely used in distributed replicated data systems. The properties of CRDTs, such as commutative operations and a semi-lattice structure, guarantee that concurrent updates can be safely executed on different replicas and later merged to get a consistent state on all replicas. The high network latency and possible reordering of messages in distributed system resulted in properties of CRDTs much different from what is required in a shared memory system. In this paper, we show implementations of mergeable data types that are tailored for shared memory concurrent programs.

Even though no consolidated theory on mergeable data types exists in the shared memory ecosystem, there have been systems that use such types with restricted properties. Doppel [15] is a multi-core database that uses a mechanism called phase reconciliation to parallelize conflicting transactions. When a high contention workload is detected, Doppel switches to a split phase where the transaction updates per-core copy of the objects. At the end of the split phase, per-core copies are merged. Only operations that are commutative are executed in the split phase, thus guaranteeing serializability. 


\section{Programming Model}

The system we consider is built upon a classical shared-memory architecture as supported by specifications such as the $\mathrm{C}++$ or Java memory models. We assume that the system consists of a variable number of threads. Any thread can spawn new threads that may outlive their parent thread. The system distinguishes two types of memory: local memory is associated to a single thread and can only be accessed by this thread; shared memory can be accessed by any thread. Communication and coordination between the threads are done via shared-memory objects; we assume that there are no side channels. In particular, spawned threads do not inherit local objects from their parents.

Each shared object $o$ has a global copy that is accessible by all threads that have a reference to it. In addition, each thread has its own local copy of $o$. A thread may update and read its own local copy, but it is not accessible by any other thread. The local updates are incorporated into the global copy when a merge operation is executed. Conflicting (non-commutative) updates from concurrent threads are resolved by a type-specific merge operation. In addition to the local updates and reads, the model also provides updates and read directly on the global copy. This gives flexibility for the data type semantics and the implementation of the underlying data structure.

An operation opKind on an object in the global-local view model can be formalized as a function

$$
\operatorname{opKind}_{t}\left(m, g, s_{t}, l_{t}\right)=\left(r, g^{\prime}, s_{t}^{\prime}, l_{t}^{\prime}\right)
$$

where $m$ comprises the (optional) type-specific update $(u)$ or query $(q) \operatorname{method}$ applied on the object, $g$ denotes the shared global object on which the operation is applied, and $t$ is a thread identifier that refers to the non-shared local version $\left(s_{t}, l_{t}\right)$ of the object. Here, $s_{t}$ denotes a local snapshot of the shared object state $g$ which gets updated upon synchronization, and $l_{t}$ refers to the local updates not yet incorporated in the shared global state $g$. The operation returns a tuple $\left(r, g^{\prime}, s_{t}^{\prime}, l_{t}^{\prime}\right)$ where $r$ is the return value of the method $m$ and the other variables refer to the updated global $g^{\prime}$ and local state $s_{t}^{\prime}, l_{t}^{\prime}$. State variables $-g, s_{t}, l_{t}-$ are each modeled as a sequence of updates, initially empty; a sequence $x$ can be concatenated with another sequence $y$ (or a single update), denoted by $x \cdot y$.

Following are the basic operations in the global-local view model; these are type-independent:

- pull updates the local object snapshot with the global object state; local operations are not changed.

$$
\operatorname{pull}_{t}\left(g, s_{t}, l_{t}\right)=\left(\perp, g, g, l_{t}\right)
$$

- weakRead returns the result of a type-specific read-only operation $q$ on the state obtained by applying local updates on the local snapshot.

$$
\text { weakRead }_{t}\left(q, g, s_{t}, l_{t}\right)=\left(q\left(s_{t} \cdot l_{t}\right), g, s_{t}, l_{t}\right)
$$

- strongRead returns the result of a type-specific read-only operation $q$ on the state obtained by applying local updates on global state. Neither the global state nor the local snapshot are changed.

$$
\operatorname{strongRead}_{t}\left(q, g, s_{t}, l_{t}\right)=\left(q\left(g \cdot l_{t}\right), g, s_{t}, l_{t}\right)
$$


- weakUpdate applies the update method $u$ on the local copy without any synchronization to the global state.

$$
\text { weakUpdate }_{t}\left(u, g, s_{t}, l_{t}\right)=\left(s_{t} \cdot l_{t} \cdot u, g, s_{t}, l_{t} \cdot u\right)
$$

- strongUpdate applies the update method $u$ on the global state atomically. The previous weak updates that are batched in $l_{t}$ are not merged at this point.

$$
\text { strongUpdate }_{t}\left(u, g, s_{t}, l_{t}\right)=\left(g \cdot u, g \cdot u, s_{t}, l_{t}\right)
$$

- merge incorporates the local updates to the global states and updates the local snapshot.

$$
\operatorname{merge}_{t}\left(g, s_{t}, l_{t}\right)=\left(\perp, g^{\prime}, g^{\prime}, \perp\right)
$$

where $g^{\prime}=\operatorname{merge}\left(g,\left(s_{t}, l_{t}\right)\right)$ and merge is type specific merge operation. In general, if the updates are commutative, $g^{\prime}=g \cdot l_{t}$. The data types can also specify a conflict resolving merge operation, in case of non-commutative concurrent updates.

While weakRead and weakUpdate act exclusively on the local copy, strongRead and strongUpdate act on the global state. The combination of these two operations supports flexible optimizations on each individual data type. For example, a queue can guarantee that an element is dequeued only once by executing dequeues in strongUpdate. At the same time, enqueues can be applied as weakUpdate and merged later for better performance. For an integer counter, we may want to enforce a weak limit on the maximum value, i.e. its value should not diverge arbitrarily from the defined maximum value. Such a counter can use a strongRead to check the global value to adapt the merge frequency or to switch to a fully synchronized version.

\section{Data Types}

Each mergeable type defines a subset of the basic operations from the globallocal view model, depending on the semantics needed. A purely mergeable counter defines only weak operations and merge, while a hybrid mergeable counter also defines strong operations. In this section, we discuss the specification of several data types and their implementation.

\subsection{Specification}

Given a sequential counter with methods inc (increments the counter by 1), value (returns the current value), a purely mergeable counter implements following operations.

- weakValue $_{t}()=$ weakRead $_{t}\left(\right.$ value $\left.,{ }_{-}, s_{t}, l_{t}\right)$

- weaklnc $_{t}()=$ weakUpdate $_{t}\left(\right.$ inc $\left.,,_{-},{ }_{-}, l_{t}\right)$

- $\operatorname{merge}\left(g,\left(s_{t}, l_{t}\right)\right)=g \cdot l_{t}$ 
The merge appends the local increments to the global sequence $g$, because the increments are commutative. A hybrid mergeable counter defines the following operations in addition to the above ones. The applications may choose weak or strong operations dynamically based on different criteria.

- $\operatorname{strong}_{\operatorname{lnc}}()=$ strongUpdate $_{t}\left(\right.$ inc $\left., g,,_{-},{ }_{-}\right)$

- strongValue $_{t}()=\operatorname{strongRead}_{t}\left(\right.$ value $\left., g,_{,}, l_{t}\right)$

A sequential queue has operations enqueue(e) and dequeue. A hybrid mergeable queue with mergeable enqueue and synchronized dequeue defines the following operations:

- enqueue $_{t}(e)=$ weakUpdate $_{t}\left(\operatorname{enqueue}(e),{ }_{-},{ }_{-}, l_{t}\right)$

- dequeue $_{t}()=$ strongUpdate $_{t}\left(\right.$ dequeue $\left., g,_{-},{ }_{-}\right)$

- $\operatorname{merge}\left(g,\left(s_{t}, l_{t}\right)\right)=g \cdot l_{t}$

In the above semantics, if the global copy is empty, dequeue returns null even if there are local enqueue operations by the same thread which have not been merged yet. We can allow dequeue to include local enqueue operations by defining

$$
\text { dequeue }_{t}()=\text { strongUpdate }_{t}\left(\text { dequeue, } g^{\prime},{ }_{-},{ }_{-}\right) \text {with }\left({ }_{-}, g_{-}^{\prime},{ }_{-}\right)=\operatorname{merge}_{t}\left(g, s_{t}, l_{t}\right) \text {. }
$$

In this way we can combine the operations to give different semantics. For example, a queue with weak enqueue and weak dequeue may be useful if redundant dequeue is not a problem for the application. A queue with both strong enqueue and strong dequeue behaves as a linearizable queue.

A grow-only bag is a set that allows only add operation, and allows duplicate elements. A purely mergeable bag implements weakAdd and merge.

\subsection{Implementation}

The implementation of (hybrid) mergeable data types consists of two parts an object variable for local view and another for global view. Local view and global view may or may not be of same type. A generic pattern for implementing a mergeable data type MDT is given by the following (object-oriented programming inspired) pseudocode:

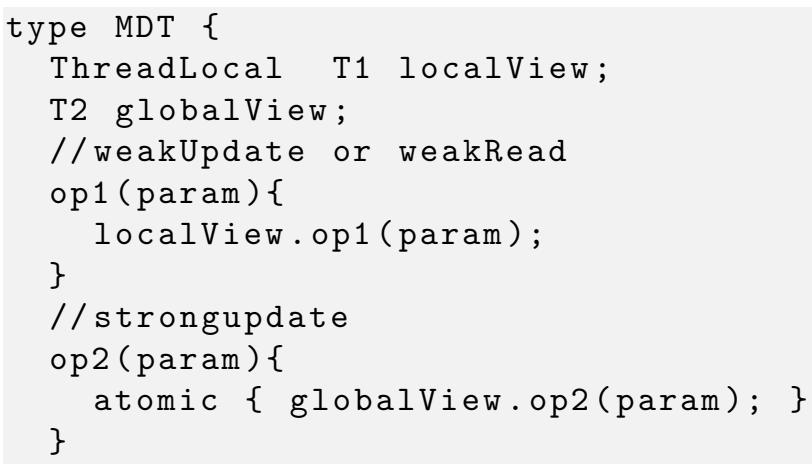




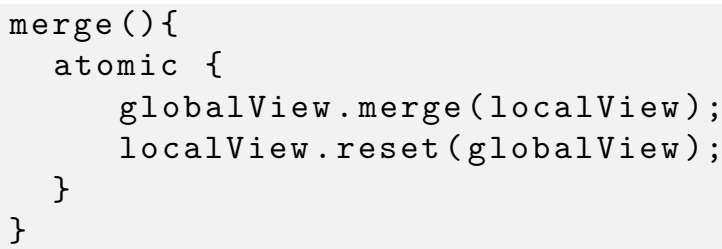

The types of localview and globalview $(\mathrm{T} 1, \mathrm{~T} 2)$ may or may not be of same type. Local views are thread-local instances as identified by ThreadLocal. A variable specified as ThreadLocal exists per thread in the thread's private storage. Many programming languages support some form of thread-local storage (TLS). A mergeable data type can also implement its own thread local storage by mapping thread ids to different instances of the object.

atomic refers to any synchronization mechanism such as mutex or lock-free techniques such as compare and swap or transactional memory that atomically executes the code block with in. op1, op2 refers to the methods implementing object's update or query operations. reset updates the local view to the global view.

weak operations are executed on the local view. The ThreadLocal descriptor guarantees that each thread is accessing its own private view. For some data types, local views are isolated from each other and the global view, by maintaining a full copy of the object in each view. For large data structures, such as list or trees, maintaining a full copy is not efficient. Thus the local views may contain references to parts of the data structures that are shared by other local views or global view. In most cases, the shared parts are not directly updated by the weak updates, but only read. For example, a lookUp on a list may first traverse the locally added items and then the shared parts of the list which are conceptually part of its local view. The mechanisms to make sure that an update on the global view does not change the local views, if it is updating the shared part, depends on the data type semantics and the underlying data structure being used. We show designs of a few data types where this can be done efficiently and correctly without copying the entire data structure.

Counter The global view of a mergeable counter is an integer $g$. The local view consists of a pair of integers $(s, l)$. The weak increments are collected in the variable $l$ and added to $g$ during the merge. This design is inspired on sloppy counters [5], while using a local counter per thread instead of per core. The following pseudocode shows the implementation of a counter.

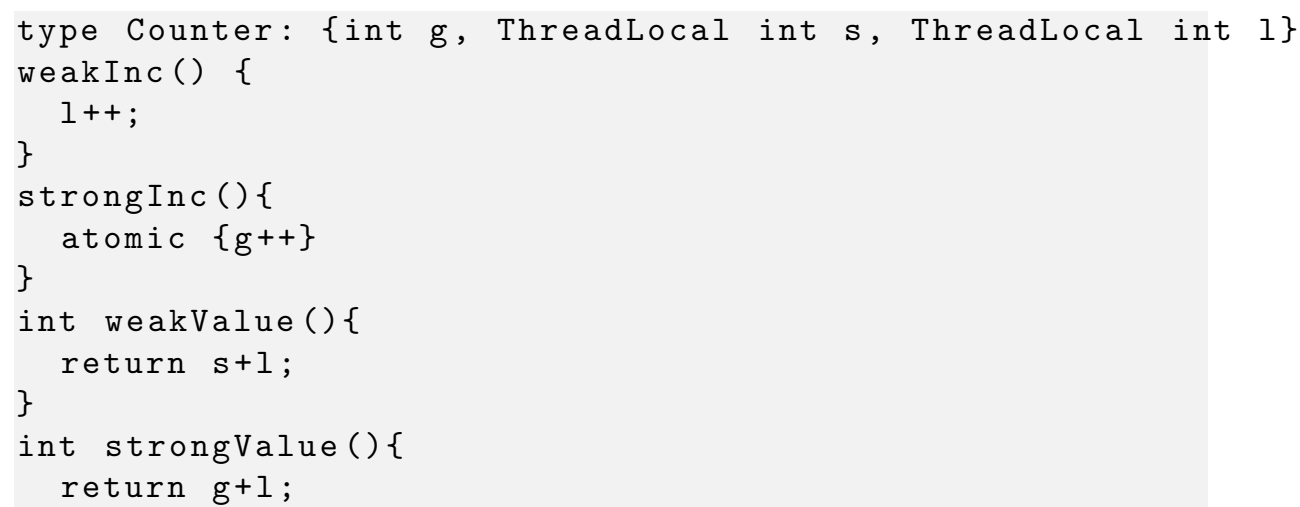




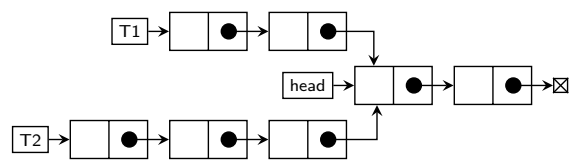

(a) Two threads with different local views.

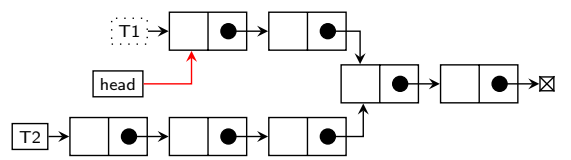

(b) After T1's local view is merged.

Figure 1: Mergeable grow-only bag.

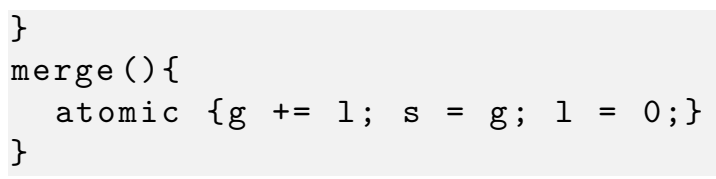

It is easy to extend this implementation to allow decrements, explicit arguments for increments/decrements, and generalize to other commutative monoids.

Grow-only bag A grow-only bag is implemented using a multi-headed list as shown in Figure 1. The thread local view consists of a pointer to the local head. A merge updates the global head of the list and does not change the local views of other threads. A lookup that traverses the list starting from the local head will never see an item that is concurrently added or merged.

Queue A hybrid mergeable queue can be implemented using a singly-linked list similar to a linearizable queue. The items enqueued are added to the tail of the list, while dequeue is performed from the head. A mergeable queue instance contains a global view - (head, tail), which points to the head and tail nodes respectively of the global list and local view - (ThreadLocal lhead, ThreadLocal 1tail), which are the head and the tail of the local list of each thread. The local list collects the items enqueued by the thread that are not yet merged. The merge atomically appends the local list to the global list (Figure 21. The time needed to merge a group of nodes is the same as the time needed to enqueue a single node. By batching the enqueues, we can reduce the number of synchronization operations, thus improving the overall throughput.

The dequeue operation directly updates the shared part of the list. For some data types, an update on the shared part of the data structure should preserve the old version, because local views may be keeping reference to it. However, there is no weakRead, such as a weak lookup, defined on queue that must observe a version before a concurrent dequeue. Hence, there is no need to keep those versions, which simplifies the implementation.

\section{Correctness Definitions}

The data types designed using the global-local view model exhibit weaker consistency than linearizability. We define the consistency model of mergeable and hybrid data types, named GLCONSISTENCY , based on the notion of abstract executions, following the formalization in [19].

An operation issued by a process on a shared object is represented by an event $e$, which is a tuple (proc, kind,type, obj, ival, oval, stime, rtime), where 


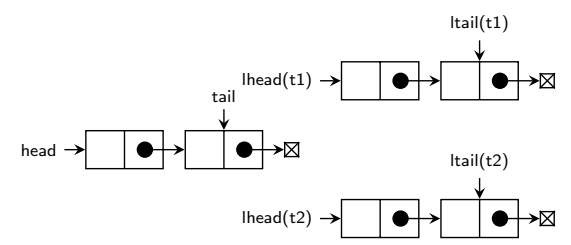

(a) Two threads with local unmerged enqueues.

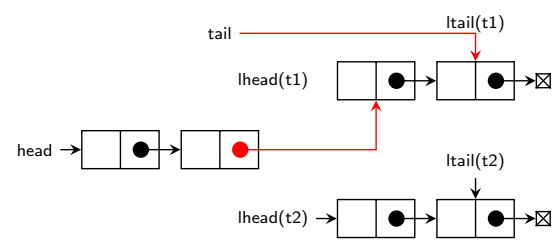

(b) Thread 1 merges its local queue.

Figure 2: Hybrid mergeable Queue.

- proc is the id of the thread issuing the event.

- kind denotes one of the operations defined in Section 3 (weakUpdate, weakRead etc.).

- type is the type-specific update or query method performed by the operation.

- $o b j$ denotes the id of the object on which the operation is performed.

- ival refers to the input parameters for the update/query method.

- oval is return value of update/query method.

- stime is the event invocation time. We assume an abstract global time that can be used to determine relative ordering of events happening in concurrent. threads.

- rtime is the event return time.

A history $H$ is a set of events. There are different relations defined on events in a history. A session order $s o$ is a partial order on the events. Two events $a, b$ are related by $s o, a \stackrel{\text { so }}{\longrightarrow} b$, if both are invoked by the same thread and $a$ returns before $b$ is invoked. For other relations, we refer to [19].

An abstract execution is a multigraph $A=(H, v i s, a r)$. vis is a partial order relation where $a \stackrel{v i s}{\longrightarrow} b$ indicates that the effects of $a$ are visible to $b$. For example, if an increment operation is visible to a read, this means that the read returns a value of the counter obtained after executing the increment. ar is a total order that specifies how concurrent operations are ordered. For example, two concurrent merge operations may be ordered based on the order of lock acquisition.

Further, the context of an event $\left.\operatorname{cxt}(A, e) \triangleq A\right|_{e, v i s^{-1}, v i s, a r}$ encodes the events prior to $e$, which may influence its return value. The specification of a data type is given by a function $\mathcal{F}$ that determines the set of intended return values of an update or a query method in relation to its context.

We extend the formalism to specify GLCONSISTENCYas follows. e.kind $\in$ $\{s u, s r, w u, w r$, pull,merge $\}$ denotes the operations strongUpdate, strongRead, weakUpdate, weakRead, pull and merge. $\left.a r\right|_{k}$ denotes the subset of $a r$ which involves only the operations where e.kinds $\in k$ and $(a, b) \in$ ar $\left.\right|_{k_{a} \rightarrow k_{b}} \Longleftrightarrow$ a.kind $\in k_{a} \wedge$ b.kind $\in k_{b} \wedge a \stackrel{a r}{\longrightarrow} b$. (Similarly, so $\left.\right|_{k}$ defines the subset of $s o$ restricted to $k$ ). 
GLConsistency is defined per object. Henceforth, to simplify the notation, we assume that a history contains only operations on a single object. We can extend the definition to include a general history by extending predicates to restrict the operations on the same object. (For example, ar $\left.\right|_{\text {merge }} \cap o b \subseteq v i s$, where $a \stackrel{o b}{\longrightarrow} b$ if $a, b$ are events applied on the same object). For a history $H$ and $\mathcal{A}$, the set of all abstract executions on $H$, we say that $H$ satisfies GLConsistency, if there exists an abstract execution $A \in \mathcal{A}$ such that $A$ satisfies the following predicate.

$$
\begin{aligned}
& \operatorname{GLCONSISTEnCy}(\mathcal{F}) \triangleq \operatorname{GLOBALORDER} \wedge \operatorname{THREAdLOCALORdER} \wedge \operatorname{ViS} \wedge \operatorname{RVAL}(\mathcal{F}) \\
& \mathrm{GLOBALORDER} \triangleq \text { ar }\left.\right|_{\text {su,merge }} \subseteq \text { vis } \wedge \text { ar }\left.\right|_{\text {su,merge } \rightarrow \text { pull }, \text { sr }} \subseteq \text { vis } \\
& \text { THREADLOCALORDER }\left.\triangleq s o\right|_{w u, w r, p u l l, m e r g e} \subseteq \text { vis } \wedge \text { so }\left.\right|_{w u \rightarrow s r} \subseteq \text { vis } \\
& \mathrm{VIS} \triangleq v i s=\left.a r\right|_{s u, m e r g e} \cup \text { ar }\left.\left.\left.\right|_{s u, \text { merge } \rightarrow \text { pull, }, s r} \cup s o\right|_{w u, w r, p u l l, m e r g e} \cup s o\right|_{w u \rightarrow s r} \\
& \operatorname{RVAL}(\mathcal{F}) \triangleq \forall o p \in H: \text { op.oval } \in \mathcal{F}(\text { op }, \operatorname{cxt}(A, \text { op }))
\end{aligned}
$$

The updates on the global copy (strongUpdate and merge) are serializable. The reads from the global copy (strongRead and pull) observe events in this order. This is defined by GlobalOrder. ThreAdLocalOrder defines the visibility of thread local operations. The visibility of a thread's operation is defined by the session order except for the strongUpdate, because strongUpdate is executing only on the global copy. However, a strongRead observes the local weakUpdates, which is captured by the predicate $\left.s o\right|_{w u \rightarrow s r} \subseteq$ vis . In addition, the visibility relation is restricted by VIs, guaranteeing that two operations from different threads are related by visibility only if there is a synchronous operation on the global view between them. (Note that this predicate is the union of the predicates from GlobalOrder and ThreadLocAlOrder).

If thread A performs weaklnc;merge, then thread B performs pull;weakValue, GLOBALORDER guarantees that thread A's merge is visible to thread B's pull which is again visible to its weakValue by THREADLOCALORDER and by transitivity weaklnc is visible to weakValue. If the thread performs a weaklnc, and then a weakValue, weaklnc is visible to weakValue. However, a stronglnc is not visible to a following weakValue, unless there is a merge or pull before the read.

RVAL is the return value consistency, which guarantees that the return value of all operations belongs to intended values based on the specification $\mathcal{F}$.

\section{Evaluation}

We evaluated the performance and scalability of the mergeable counter and the hybrid mergeable queue using different micro-benchmarks. As an example of real applications, we employed the hybrid queue in a breadth-first traversal on graphs. We implemented the counter in $\mathrm{C}++$ and the queue in Java.

The evaluations are performed on a 12 core $2.40 \mathrm{GHz} \operatorname{Intel}(\mathrm{R}) \mathrm{Xeon}(\mathrm{R})$ CPU E5-2620 processor (2 NUMA nodes) with 2-way hyper-threading, under linux 4.4.0-62 Ubuntu x86_64 and openjdk version 1.8.0_121, clang version 4.0.0svn297204-1, boost 1.58.0.1ubuntu1. 


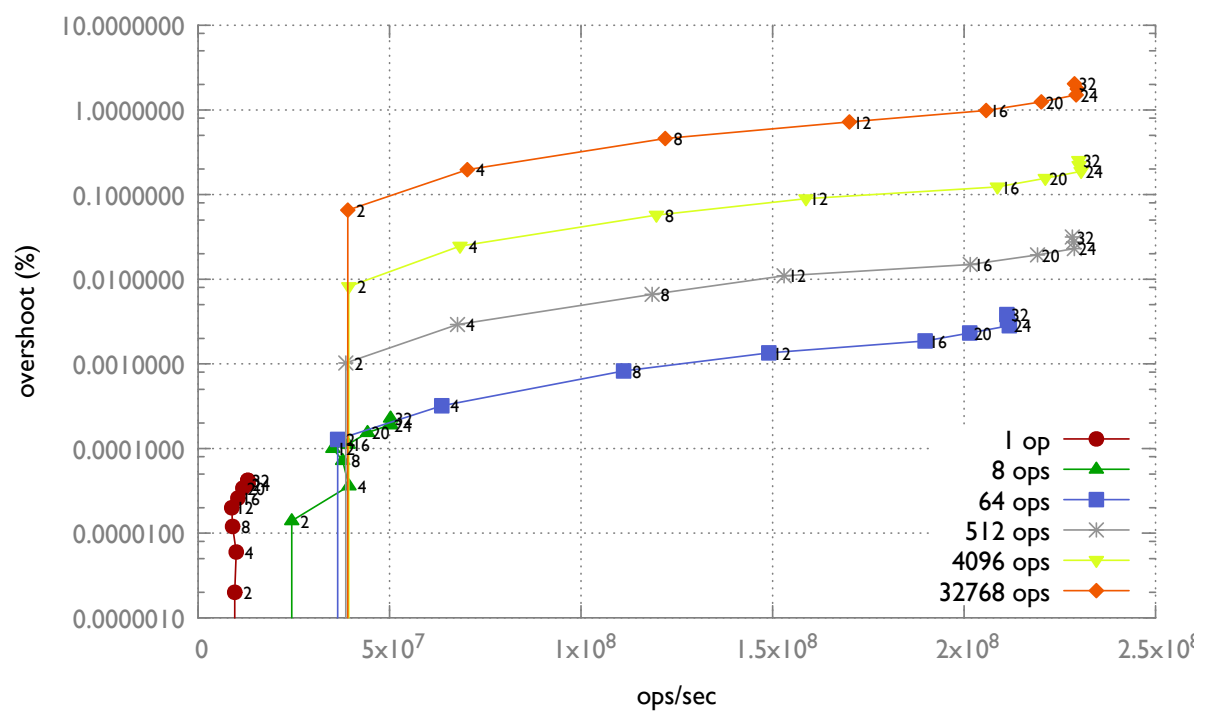

Figure 3: Throughput vs Overshoot of mergeable counter with different merge frequency.

Counter We provide two variants of a mergeable counter and compare them with an atomic counter, implemented using the atomic compare and swap operation. The first version implements a weak mergeable counter, and is based on making threads increment their local count and periodically merge with the global count, also using atomics for efficiency. In the experiment, we allow threads to increment the shared mergeable counter until a target value is reached. Since threads might not know about non-merged increments from other threads, they typically end up overshooting the target. For this experiment, the target is set to $5 \times 10^{6}$ increments. We evaluated several merge frequencies, labeled with how many local increments are allowed between merges, and measured their throughput and the overshoot from the target. Figure 3 shows that the throughput scales with the number of threads and with the merge frequency. At the same time, the overshoot increases. However, the percentage of the overshoot is small. (Notice that overshoot is upper bound by the number of threads multiplied by the merge frequency, as this reflects at any given time the amount of increments not yet accounted for.) Points in the lines are labeled with the number of threads used. As expected, the system does not scale beyond the point where the number of threads exceeds the number of cores (i.e at 24 threads). Also, note that for a single thread, overshoot is zero and thus the value is outside the logarithmic scale.

Figure 4 shows the throughput of the mergeable counter compared to an atomic counter implemented using compare and swap. The atomic counter never overshoots the target, but since threads are always competing on the increment, performance is very low and no speedup is obtained from multi-threading. In contrast, the mergeable counter can scale linearly up to a good fraction of the available concurrency, in particular with merge frequency of $\geq 4096$.

While some applications could tolerate an overshoot, in general, applications will require a tight target enforcement. To address this, we provide a variant 


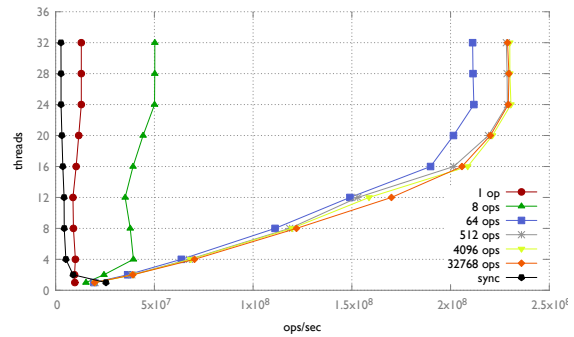

Figure 4: Throughput of mergeable counter vs atomic counter.

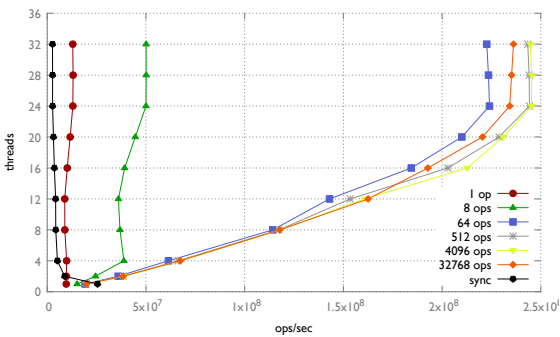

Figure 5: Throughput of hybrid mergeable counter (overshoot free) vs atomic counter.

of the mergeable counter that makes a hybrid use of initial weak local increments and later switches to atomic strong increments when approaching the target. The first thread that, upon the periodic merges, detects that it is close to the target, initiates a barrier synchronization to ensure that all threads have switched to strong operations. Figure 5 shows that under this approach, overshoot is eliminated while the performance is mostly identical to the mergeable counter. In general, the hybrid approach is efficient as long as the target is much larger than the merge frequency, since this limits the proportion of the execution done under linearizability.

Queue To evaluate the scalability of hybrid mergeable queue (referred to as mergeable queue), we implemented four different queues in Java - 1) a lockbased linearizable queue based on Michael and Scott's 2-lock queue [14, 2) a lock-based mergeable queue which uses similar 2-lock mechanism, 3) a lock-free linearizable queue adapted from Michael and Scott's lock-free queue 14 and 4) a lock-free mergeable queue. We evaluated the time to do a total of $5 \times 10^{6}$ enqueues and dequeues. Figure 6 shows the result, evaluating mergeable queues with different merge frequencies $m$ (a merge is performed by a thread after $m$ enqueues). In this experiment, we forced half of the threads to run on one NUMA node and the other half on the second NUMA node. For both lockbased and lock-free versions, the mergeable queue is faster than the linearizable counterpart. Since this is a high-contention workload, the lock-based version performs better than the lock-free version. Unlike the mergeable counter, increasing merge frequency from 8 to 64 does not improve the performance significantly. The reason is that, dequeue is always executed synchronously which shadows the performance gain from asynchronous enqueues.

Breadth-First Traversal A standard breadth-first traversal algorithm using queues can be parallelized using concurrent queues. We evaluated four versions of the algorithm using different queue implementations, that traversed randomly generated graphs of size of $2 \times 10^{6}$ vertices and $2 \times 10^{7}$ edges. Unlike the micro-benchmark for the queue, there is no fixed merge frequency. The threads merge their local queue at the end of processing each level. Figure 7 shows the speedup of each version compared to a single-threaded implementation. Mergeable queues scale better than their linearizable counterparts. The speedup of the lock-free mergeable queue is significantly higher than that of the others, 


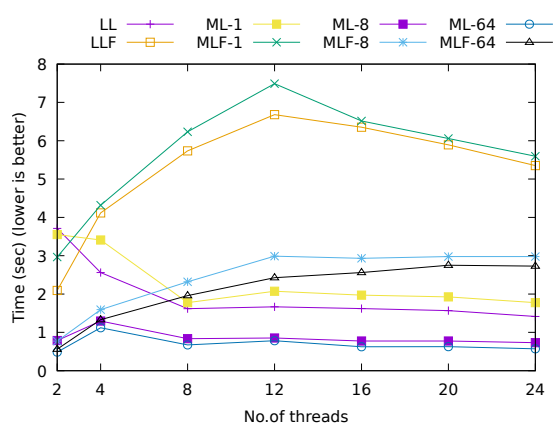

Figure 6: Queue. LL: linearizable lock-based, LLF: linearizable lock-free, ML: mergeable lock-based, MLF: mergeable lock-free. 1,8,64 - merge frequency for mergeable queues.

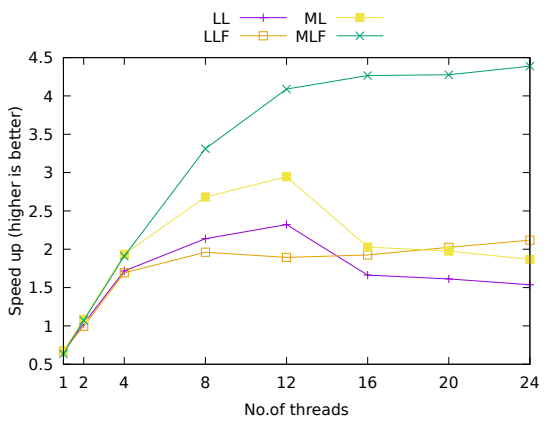

Figure 7: Breadth-first traversal on a graph using different queue implementations. LL: linearizable lockbased, LLF: linearizable lock-free, ML: mergeable lock-based, MLF: mergeable lock-free.

and scales almost linearly until 16 threads. Beyond 16 threads, the number of vertices processed by each thread at each level is reduced, as they are divided among the threads, leading to smaller merge frequencies. We believe the sudden drop in the speedup of lock-based queues after 12 threads is due to the additional cost in synchronization to the second NUMA core. Compared to the highcontention micro-benchmark from Figure 6, this is a low-contention workload because a significant amount of time is spent in processing the nodes rather than updating the queue.

\section{Conclusion}

An ever-increasing number of cores in combination with heterogeneous access latencies at different cache levels have advanced the spectrum of attainable performance from multi-thread programming. At the same time, this breaks the transparency with respect to data locality. As processor components become more numerous and spatially distributed, the cost of synchronization and communication among distant components will keep increasing in comparison to ones that are more closely located. When building internet-scale distributed systems, similar concerns lead to the design of scalable systems that limit global synchronization and operate locally when possible [12.

Incorporating more information about the respective datatype semantics is crucial for datatype designs that are more parsimonious regarding synchronization. CRDTs succeed in capturing datatypes with clear concurrency semantics and are now common components in internet-scale systems. However, they do not migrate trivially to shared-memory architectures due to high computational costs from merge functions, which becomes apparent once network communication is removed.

In this paper, we define the global-local view model as base for a framework that allows capturing the semantics of multi-view datatypes. The global-local 
view distinguishes between local fast state and distant shared state where operations need to be synchronized. This distinction allows the datatype designer to explore the trade-offs in the design when using weak or strong operations. Our approach enables speedups in order of magnitudes while preserving the datatypes' target behavior. We believe that the examples shown here are just the tip of the iceberg in terms of applicable datatypes. It is quite possible that further increments of the number of components involved will lead to a multi-tier model with more levels than the current binary, local vs global, scheme.

\section{References}

[1] Yehuda Afek, Guy Korland, and Eitan Yanovsky. Quasi-linearizability: Relaxed consistency for improved concurrency. In Proceedings of the 14th International Conference on Principles of Distributed Systems, OPODIS'10, pages 395-410, Berlin, Heidelberg, 2010. Springer-Verlag.

[2] Amitanand Aiyer, Lorenzo Alvisi, and Rida A. Bazzi. On the availability of non-strict quorum systems. In Proceedings of the 19th International Conference on Distributed Computing, DISC'05, pages 48-62, Berlin, Heidelberg, 2005. Springer-Verlag.

[3] Deepthi Devaki Akkoorath and Annette Bieniusa. Transactions on mergeable objects. In Xinyu Feng and Sungwoo Park, editors, Programming Languages and Systems - 13th Asian Symposium, APLAS 2015, Pohang, South Korea, November 30 - December 2, 2015, Proceedings, volume 9458 of Lecture Notes in Computer Science, pages 427-444. Springer, 2015.

[4] Deepthi Devaki Akkoorath and Annette Bieniusa. Highly-scalable concurrent objects. In Proceedings of the $2 N d$ Workshop on the Principles and Practice of Consistency for Distributed Data, PaPoC '16, pages 13:1-13:4, New York, NY, USA, 2016. ACM.

[5] Silas Boyd-Wickizer, Austin T. Clements, Yandong Mao, Aleksey Pesterev, M. Frans Kaashoek, Robert Morris, and Nickolai Zeldovich. An analysis of linux scalability to many cores. In Proceedings of the 9th USENIX Conference on Operating Systems Design and Implementation, OSDI'10, pages 1-16, Berkeley, CA, USA, 2010. USENIX Association.

[6] Sebastian Burckhardt, Alexandro Baldassin, and Daan Leijen. Concurrent programming with revisions and isolation types. In Proceedings of the ACM International Conference on Object Oriented Programming Systems Languages and Applications, OOPSLA '10, pages 691-707, New York, NY, USA, 2010. ACM.

[7] Sebastian Burckhardt, Alexey Gotsman, Hongseok Yang, and Marek Zawirski. Replicated data types: Specification, verification, optimality. In Proceedings of the 41st ACM SIGPLAN-SIGACT Symposium on Principles of Programming Languages, POPL '14, pages 271-284, New York, NY, USA, 2014. ACM.

[8] Sebastian Burckhardt, Daan Leijen, Jonathan Protzenko, and Manuel Fähndrich. Global Sequence Protocol: A Robust Abstraction for Replicated 
Shared State. In John Tang Boyland, editor, 29th European Conference on Object-Oriented Programming (ECOOP 2015), volume 37 of Leibniz International Proceedings in Informatics (LIPICs), pages 568-590, Dagstuhl, Germany, 2015. Schloss Dagstuhl-Leibniz-Zentrum fuer Informatik.

[9] Thomas E. Hart, Paul E. McKenney, and Angela Demke Brown. Making lockless synchronization fast: Performance implications of memory reclamation. In Proceedings of the 20th International Conference on Parallel and Distributed Processing, IPDPS'06, pages 21-21, Washington, DC, USA, 2006. IEEE Computer Society.

[10] Maurice P. Herlihy and Jeannette M. Wing. Linearizability: A correctness condition for concurrent objects. ACM Trans. Program. Lang. Syst., 12(3):463-492, July 1990.

[11] Alex Kogan and Maurice Herlihy. The future(s) of shared data structures. In Proceedings of the 2014 ACM Symposium on Principles of Distributed Computing, PODC '14, pages 30-39, New York, NY, USA, 2014. ACM.

[12] Cheng Li, Daniel Porto, Allen Clement, Johannes Gehrke, Nuno Preguiça, and Rodrigo Rodrigues. Making geo-replicated systems fast as possible, consistent when necessary. In 10th USENIX Symposium on Operating Systems Design and Implementation (OSDI 12), pages 265-278, Hollywood, CA, 2012. USENIX.

[13] Alexander Matveev, Nir Shavit, Pascal Felber, and Patrick Marlier. Readlog-update: A lightweight synchronization mechanism for concurrent programming. In Proceedings of the 25th Symposium on Operating Systems Principles, SOSP '15, pages 168-183, New York, NY, USA, 2015. ACM.

[14] Maged M. Michael and Michael L. Scott. Simple, fast, and practical nonblocking and blocking concurrent queue algorithms. In Proceedings of the Fifteenth Annual ACM Symposium on Principles of Distributed Computing, PODC '96, pages 267-275, New York, NY, USA, 1996. ACM.

[15] Neha Narula, Cody Cutler, Eddie Kohler, and Robert Morris. Phase reconciliation for contended in-memory transactions. In Proceedings of the 11th USENIX Conference on Operating Systems Design and Implementation, OSDI'14, pages 511-524, Berkeley, CA, USA, 2014. USENIX Association.

[16] Marc Shapiro, Nuno Preguiça, Carlos Baquero, and Marek Zawirski. Conflict-free replicated data types. In Proceedings of the 13th International Conference on Stabilization, Safety, and Security of Distributed Systems, SSS'11, pages 386-400, Berlin, Heidelberg, 2011. Springer-Verlag.

[17] Nir Shavit. Data structures in the multicore age. Commun. ACM, 54(3):7684, March 2011.

[18] Martin Sstrik. Zeromq. In The Architecture of open source applications, Volume 2, 2012.

[19] Paolo Viotti and Marko Vukolić. Consistency in non-transactional distributed storage systems. ACM Comput. Surv., 49(1):19:1-19:34, June 2016. 